Classification

Physics Abstracts

$68.10-75.50 \mathrm{~B}-75.50 \mathrm{M}$

\title{
Instability of ferrofluid magnetic drops under magnetic field
}

\author{
J. C. Bacri and D. Salin \\ Laboratoire d'Ultrasons $\left({ }^{*}\right)$, Université Pierre et Marie Curie, Tour 13, \\ 4, place Jussieu, 75230 Paris Cedex 05, France
}

(Reçu le 10 juin 1982, accepté le 5 juillet 1982)

\begin{abstract}
Résumé. - Nous avons suivi l'évolution de la forme de gouttes magnétiques de ferrofluide plongées dans un champ magnétique. La forme ellipsoïdale allongée de la goutte devient instable pour un certain champ magnétique seuil : la goutte passe d'une forme peu allongée à une forme beaucoup plus allongée. En baissant le champ magnétique, le même phénomène se produit mais pour une valeur de seuil plus faible. Cette instabilité est interprétée à partir des énergies magnétique et interfaciale de la goutte.
\end{abstract}

\begin{abstract}
We have followed the evolution of the shape of ferrofluid magnetic drops in presence of a magnetic field. The prolate ellipsoid shape of the drop becomes unstable for a certain magnetic field threshold : the drop jumps from a slightly elongated shape to a much more elongated shape. When decreasing the magnetic field the same feature occurs for a smaller threshold. This instability is simply understood from a balance between magnetic energy and interfacial tension energy.
\end{abstract}

1. Introduction. - Since 1925 [1-3], it is well known that when a fluid containing an immiscible fluid drop is subjected to an electric field, the drop deforms into a prolate ellipsoid whose axis of rotation is along the field direction. When the field is increased up to a certain threshold the drop becomes unstable and bursts [4,5]. For a perfect dielectric, the deformation into a prolate spheroid and the subsequent burst of the drop is due to a competition between electric energy which favours an elongated shape in the field direction and surface energy which favours a spherical shape [3, 4]. Analogous deformations have been observed for an immiscible drop subjected to an extensional hydrodynamic flow $[6,7]$ with an hydrodynamic stress in place of the electric one.

Analogy between electric and magnetic properties predicts similar behaviour for an immiscible magnetic fluid drop in a fluid [8-10]. Recent developments in the manufacture of ferrofluids [11] give a wide range of magnetic fluids to test the analogy. Ferrofluids are colloidal suspensions of ferromagnetic grains of a typical size of $100 \AA$. In presence of a magnetic field, agglomeration of grains occurs [12,13]. These agglomerates have been first observed as elongated needles [13] and their shape in presence of a magnetic field have recently been studied $[14,15]$. We report here on a study of the deformation and instabilities of ferrofluid agglomerate drop in a magnetic field. As we will see later on, the magnetic situation in our ferrofluid is more demonstrative than the electrostatic one.

$\left(^{*}\right)$ Associated with the Centre National de la Recherche Scientifique. 


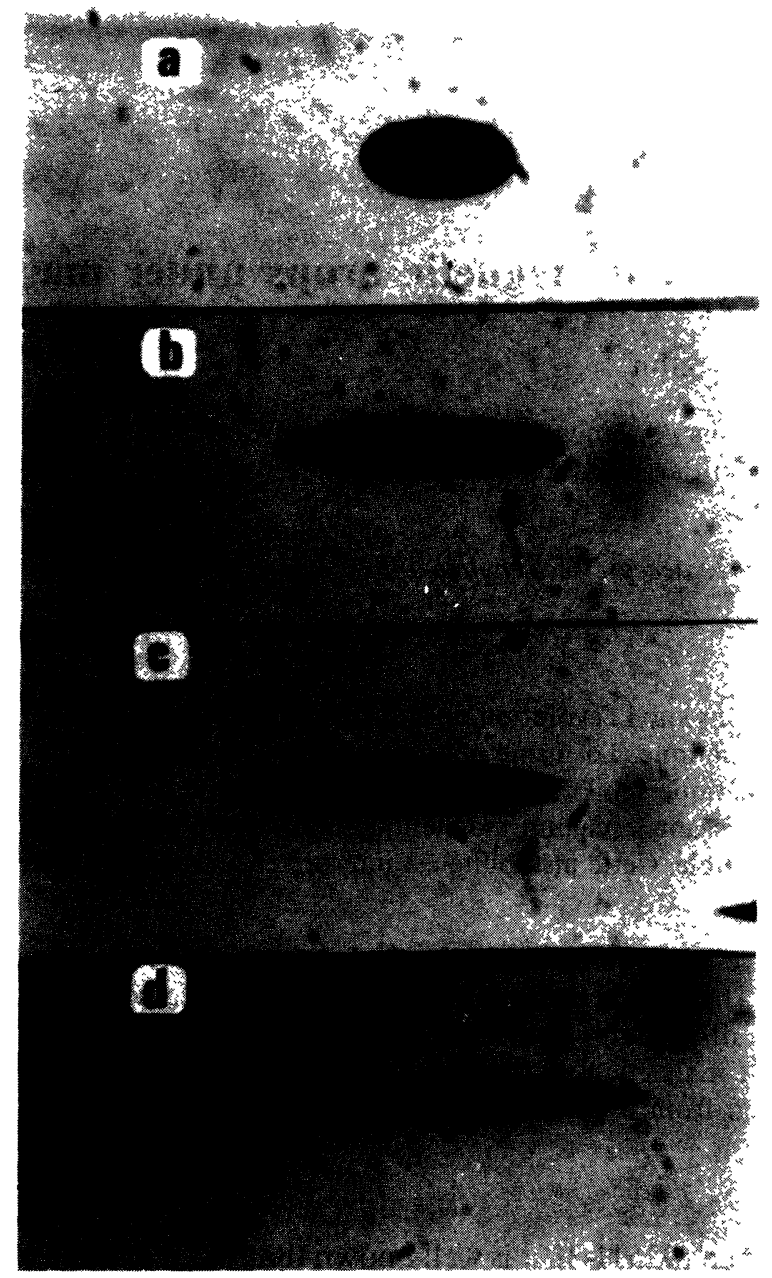

Picture 1. - The four pictures correspond to the same threshold field $\left.H_{1} . a\right) H$ was just increased to $H_{1}$; b) $1 \mathrm{~min}$. later ; c) $1 / 4 \mathrm{~min}$. later ; d) $1 \mathrm{~min}$. later : stabilized shape.

2. Theory. - The shape of a magnetic incompressible fluid drop of permeability $\mu_{2}$ in a fluid of permeability $\mu_{1}$ is due to a competition between its magnetic energy $E_{\mathrm{M}}$ and its interfacial energy $E_{\mathrm{S}}$. For two fluids of the same density the shape of the drop is usually assumed as prolate ellipsoidal. In fact the exact shape, numerically computed from basic equations [4], is very close to prolate ellipsoid, except when the spheroid becomes too much elongated. This is the experimentally observed shape where the ends of the elongated spheroid are too sharp (Pictures $1 a$ and $1 d$ ). We will use this prolate ellipsoid shape which gives analytic expression for magnetic energy. For a prolate spheroid of semi major axis $a$ and semi minor axis $b$, the surface energy is

$$
E_{\mathrm{S}}=\sigma 2 \pi a^{2} K\left[K+\varepsilon^{-1} \sin ^{-1} \varepsilon\right]
$$

where $\sigma$ is the interfacial tension, $K=b / a$ the aspect ratio, and $\varepsilon=\left(1-K^{2}\right)^{1 / 2}$ the eccentricity. If $\mu_{1}$ and $\mu_{2}$ are independent of the field (low field : magnetization $M$ much smaller than the saturated magnetization; in our ferrofluid agglomerates $M_{\mathrm{s}} \sim 400 \mathrm{G}$ and $M \lesssim 5 \mathrm{G}$ for 
$H \sim 1 \mathrm{G})$, the magnetic energy takes, in c.g.s. units, the form [16] :

$$
E_{\mathrm{M}}=-\frac{V H^{2} \mu_{1}}{8 \pi} \frac{1}{\alpha+n}
$$

$H$ is the applied magnetic field, $V=\frac{4 \pi}{3} a b^{2}$ is the volume of the ellipsoid, $\alpha=\mu_{1} /\left(\mu_{2}-\mu_{1}\right)$ and $n=K^{2}\left(-2 \varepsilon+\ln \left[(1+\varepsilon) /\left(1-\frac{\varepsilon}{\varepsilon}\right)\right]\right) / 2 \varepsilon^{3}$ is the shape dependent demagnetization factor [16] $\left(l=4 \pi n\right.$ in Refs. $[14,15] ; n=\frac{1}{3}$ for a sphere). For a constant volume $V=\frac{4}{3} \pi R_{0}^{3}\left(R_{0}\right.$ radius of the spherical drop in zero field) and a given magnetic field $H$, the shape of the drop $(b / a=K)$ is given by minimization of the total energy $E_{\mathrm{T}}=E_{\mathrm{M}}+E_{\mathrm{S}}$ with respect to the aspect ratio $K$. We get

$$
H^{2} R_{0} / \sigma=g(K)
$$

with

$$
\begin{aligned}
g(K)=\frac{8 \pi}{\mu_{1}}(n+\alpha)^{2} \varepsilon^{2} K^{-4 / 3}\left(1+2 K^{2}+(1-\right. & \left.\left.4 K^{2}\right) \varepsilon^{-1} K^{-1} \sin ^{-1} \varepsilon\right) \times \\
& \times\left(-6+\left(2+K^{2}\right) \varepsilon^{-1} \ln \frac{1+\varepsilon}{1-\varepsilon}\right)^{-1} .
\end{aligned}
$$

An expression similar to (3) is given in reference [15] for a different form of the magnetic energy $E_{\mathrm{M}}^{\prime}$. A ferrofluid colloidal suspension is an assembly of permanent magnetic dipoles. For small concentration of dipoles the suspension behaves as a paramagnetic medium and its magnetization follows Langevin equation [17]. In our agglomerates of ferrofluid the concentration in magnetic grains is large and this phase rich in grains is described [14] as an assembly of magnetic dipoles in a cubic array. The magnetic energy $E_{M}^{\prime}$ of interaction of this cubic array in presence of a magnetic field is, at low field and omitting an additive constant [14] :

$$
E_{\mathrm{M}}^{\prime}=-\frac{V H^{2}}{8 \pi} \frac{1}{\frac{Z}{4 \pi}-\frac{1}{3}+n}
$$

The number $Z$ depends on the temperature and on the structure of the cubic array. Minimization of $E_{\mathrm{T}}$ gives the same expression as (3) with the function $g(K)$ with $\mu_{1}=1$ and $\alpha=\frac{Z}{4 \pi}-\frac{1}{3}$. At $T=0$, for a simple cubic lattice, $Z=5.352$ [18] and $\alpha=0.093$. These two approaches of the problem lead to the same prediction on agglomerate shape behaviour. The value of $\alpha$ is related to the value of $\mu_{2} / \mu_{1}$. If the agglomerate is assumed solid like as in [14] $\alpha$ is related to the structure number $Z$.

Figure 1 gives a log-log plot of $a / b=K^{-1}$ versus $g(K)$ for different values of the parameter $\alpha\left(\mu_{1}=1\right)$. One can see that as $\alpha$ decreases, $g(K)$ goes from a monotonic curve to a S-shape one. The transition between these two shapes occurs for $\alpha \simeq 0.055$. In the case of a S-shape curve, for certain values of $g(K)$ (or $H^{2} R_{0} / \sigma$ through Eq. (3)) there are three values of $K$ which are extrema of $E_{\mathrm{T}}$. Straightforward calculations show that the AC part of the curve (Fig. 1), corresponds to a maximum of total energy while other parts of the curve correspond to minima of $E_{\mathrm{T}}$ : the $\mathrm{AC}$ part of the curve is unstable and points $\mathrm{A}$ and $\mathrm{C}$ are the thresholds of instability. For the two minima (with the same value of $H^{2} R_{0} / \sigma$ ), the minimum corresponding to the smallest value of $E_{\mathrm{T}}$ is stable while the other one is metastable. Points I and $\mathrm{J}$ (Fig. 1) correspond to the value of $H^{2} R_{0} / \sigma$ where the two minimum energies are equal : values of $E_{\mathrm{T}}$ on the IA (resp. JC) part of the curve are larger than the corresponding values of $E_{\mathrm{T}}$ on JB (resp. ID). From this S-shape one can expect for a cycle in magnetic field : as $H$ increases from zero, $K^{-1}=a / b$ 


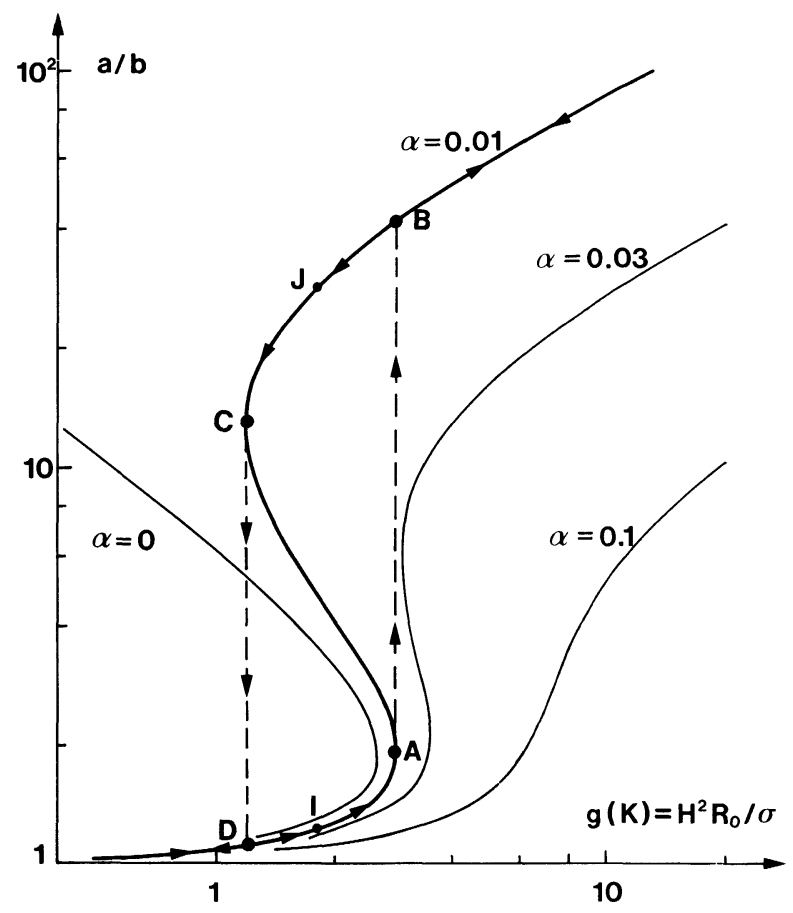

Fig. 1. - Log-log plot of the variation of $a / b=K^{-1}$ versus $H^{2} R_{0} / \sigma$ for different values of the parameter $\alpha$ (see text).

increases from 1 up to point $\mathrm{A}\left(K_{\mathrm{A}}^{-1} \sim 2\right)$. At this point $\mathrm{A}$, the metastable shape of the drop becomes unstable and it must go to the stable shape (point B) much more elongated $\left(K_{\mathrm{B}}^{-1}\right)$. Increasing $H$ again, $K^{-1}$ increases again. When the field is decreased, $K^{-1}$ first decreases down to point $\mathrm{C}\left(K_{\mathrm{C}}^{-1}\right)$. At this point $\mathrm{C}$ the metastable elongated shape of the drop becomes unstable and it must go to the stable shape (point D) less elongated $\left(K_{\mathrm{D}}^{-1}\right)$. Decreasing $H$ again to zero, $K^{-1}$ decreases to 1 . Thus for certain values of the ratio $\mu_{2} / \mu_{1}$, the deformation of a fluid magnetic drop exhibits a first order transition with hysteresis effects. Finally the same features of instabibility are possible in electrostatics for perfect dielectrics, but if the media (1 and 2) are not perfect dielectrics, the problem is much more complicated because it is not possible to neglect conductivity effects $(\alpha \rightarrow 0)$. Only one instability is observed (point $\mathrm{A}$ ).

3. Experiment. - The preparation of ferrofluids agglomerates and our experimental set up has already been described [15] : the nearly spherical $(H=0)$ agglomerates consist of a more concentrated (in magnetic grains) phase in a less concentrated phase. Their radii are between 2 and $20 \mu \mathrm{m}$. The microscope observation has been improved with a video camera and a television screen which allow us to follow the evolution of the shape of agglomerates. The accuracy in field measurement is better than $1 \%$. For each agglomerate, we use the same procedure : starting from $H=0$ (spherical shape) we increase the magnetic field by small steps. For each value of $H$ we wait until the shape is stabilized (a few minutes close to the thresholds) and we measure $a$ and $b$. After reaching a certain maximum value of $H$ (a few Gauss) we decrease the field by small steps down to zero. The variations of $a / b$ versus the square of the magnetic field $\left(H^{2}\right)$ are given in figure 2 for a $R_{0}=15 \mu \mathrm{m}$ agglomerate with different symbols for increasing $(+)$ and decreasing $(O)$ field. These experimental data clearly show the existence of two field thresholds : one for increasing field $\left(H_{1}=1.19 \mathrm{G}\right)$ and the other one for decreasing field $\left(H_{2}=1.05 \mathrm{G}\right)$. 


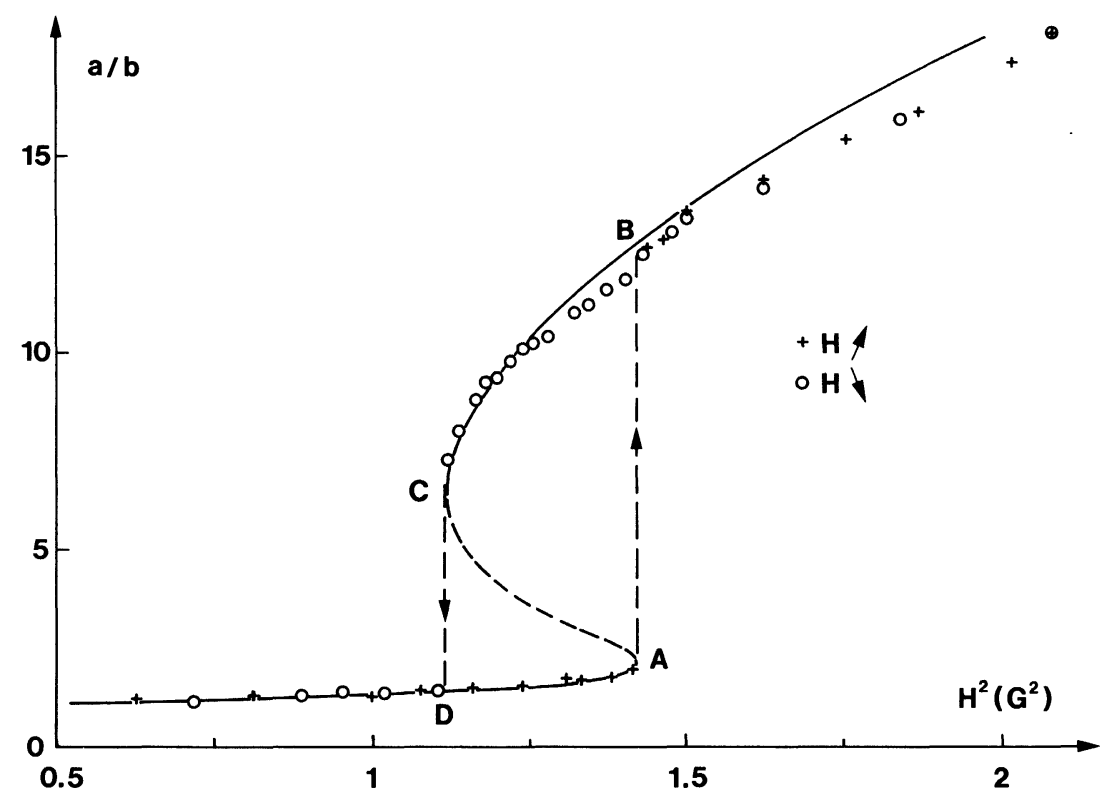

Fig. 2. - Variation of the elongation $a / b$ of the agglomerate drop versus the square of the magnetic field : (+) $H$ increasing, (O) $H$ decreasing.

At the first threshold $H_{1}$, the evolution in time of the shape of the drop is given in picture 1 by four successive pictures :

1.a) The field has just been increased at the threshold value $H_{1}$ (point $\mathrm{A}$ ), the elongation of the agglomerate is $a / b=K_{\mathrm{A}}^{-1} \simeq 2$.

1.b) $\sim 1 \mathrm{~min}$. later the instability starts to develop at one end of the drop.

1.c) A quarter minute later the other end elongates.

1.d) $\sim 1$ min. later the final stabilized shape of the $\operatorname{drop}\left(H=H_{1}\right.$, point $\left.\mathrm{B}, a / b=K_{\mathrm{B}}^{-1} \simeq 12.7\right)$.

An analog feature is observed at threshold $\mathrm{H}_{2}$ in decreasing field where agglomerate resorbs its elongated ends.

As the qualitative features of the theory are observed, we have to test it quantitatively. Theoretical fit to the data requires three constants : the interfacial tension $\sigma$, the permeabilities $\mu_{1}$ and $\mu_{2}$ of the two media. As the S shape of $g(H)$ (Fig. 1) changes rapidly with $\alpha=\left(\frac{\mu_{1}}{\mu_{2}}-1\right)^{-1}$, the experimental values of $K_{\mathrm{B}}, K_{\mathrm{C}}$, and ratio $\mathrm{H}_{1} / \mathrm{H}_{2}$ fix precisely the value of $\alpha$. Then from the absolute value of $H_{1}$ (or $H_{2}$ ) we get $\sigma / \mu_{1}$. For our $15 \mu \mathrm{m}$ agglomerate the theoretical curve in figure 2 corresponds to $\alpha=0.0265$ and $\sigma / \mu_{1}=6.5 \times 10^{-4}$ c.g.s. The accord between theory and experiment is surprisingly good except for large values of $a / b$ where agglomerate shape is less ellipsoidal. The value of $\alpha$ gives a value of $\mu_{2} / \mu_{1}=39$. If we assume that the lower density, in grains, phase is close to pure water $\left(\mu_{1} \sim 1\right)$, we get $\mu_{2} \simeq 40$. This value is high for a colloidal suspension of magnetic grains and corresponds to a very concentrated ferrofluid phase [11]. Such a concentrated phase can also be described with the structure number $Z=4.5$ of a cubic array of dipoles. The value of $\sigma \sim 6.5 \times 10^{-4}$ c.g.s. is of the same order of magnitude as in previous measurements in the same kind of ferrofluids [15] and two orders of magnitude larger than in another kind of ferrofluids [14].

Finally if each agglomerate exhibits a S-shape curve as in figure 2, the precise value of $\alpha$ deduced 
from this curve is different from one agglomerate to the other (from 0.022 to 0.029 ). Similarly the value of $\sigma / \mu_{1}$ varies from $4.5 \times 10^{-4}$ to $9 \times 10^{-4}$ c.g.s. These differences from one agglomerate to the other are most likely due to differences in concentration : $\mu_{2}$ and $\sigma$ are concentration dependent and the method of preparation of the agglomerates [15] gives agglomerates of different sizes which imply different internal pressures and therefore different values of $\mu_{2}$ and $\sigma$. This distribution of $\sigma$ and $\alpha$ values explains why the fit to the data, in our previous work [15], for nine different agglomerates together give a smooth curve showing no instability. In fact for each individual agglomerate there was the instability described here.

4. Conclusion. - Following the evolution of the shape of magnetic ferrofluid drop in presence of a magnetic field, we have observed that the shape is unstable for certain elongation values : for increasing magnetic field the drop jumps from slightly elongated $(a / b \sim 2)$ to a much more elongated shape $(a / b \sim 13)$ at a field threshold $H_{1}$. For decreasing magnetic field the elongated shape $(a / b \sim 7)$ resorbed to a less elongated one $(a / b \sim 1.5)$ at a smaller magnetic field threshold $\mathrm{H}_{2}$. These features are understood from a balance between a magnetic energy of a prolate ellipsoid and an interfacial energy.

Acknowledgments. - We are greatly indebted to Dr. Massart for providing us with ferrofluid.

\section{References}

[1] Wilson, C. T. R. and Taylor, G. I., Proc. Cambridge Philos. Soc. 22 (1925) 728.

[2] Buchner, E. H. and van Royen, A. H. H., Kolloid Z. 49 (1929) 249.

[3] O'Konski, C. T. and Thacher, H. C., J. Phys. Chem. 57 (1953) 955.

[4] Garton, C. G. and Krasucki, Z., Proc. R. Soc. London A 280 (1964) 211.

[5] TAYlor, Sir Goeffrey, Proc. R. Soc. London A 280 (1964) 383.

[6] TAYlor, G. I., Proc. R. Soc. London A 138 (1932) 41.

[7] TAYlor, G. I., Proc. R. Soc. London A 146 (1934) 501.

[8] Arkhipenko, V. I., Barkov, Yu. D. and Bashtovoi, V. G., Magnetohydrodynamics 14 (1978) 373.

[9] Arkhipenko, V. I., Barkov, Yu. D. and Bashtovol, V. G., Magnetohydrodynamics 16 (1980) 228.

[10] Drozdova, Skrobotova and Chekanov, Magnetohydrodynamics 15 (1979) 12.

[11] Massart, R., IEEE Trans. Magn. 17 (1981) 1241.

[12] Peterson, E. A. and Krueger, D. A., J. Colloid Interface Sci. 62 (1977) 24.

[13] HAYes, C. G., J. Colloid Interface Sci. 52 (1975) 239.

[14] Liao, W. H. and KRUEGer, D. A., J. Colloid. Interface Sci. 70 (1979) 564.

[15] BaCri, J. C., Salin, D. and Massart, R., J. Physique-Lett. 43 (1982) L-179.

[16] Stratton, J. D., Electromagnetic Theory (McGraw Hill Co, New-York) 1941.

[17] General reference in Shlomis, M., Sov. Phys. Usp. 17 (1974) 153.

[18] Luttinger, J. M. and Tisza, L., Phys. Rev. 70 (1946) 954. 